\title{
A Case Report of Spontaneous Pregnancy in a Mosaic Turner Syndrome Patient
}

\author{
Abdelrahman Yousif ${ }^{*}$, Madeline Wheatley ${ }^{2}$ and Moustafa A Abuzeid ${ }^{1}$ \\ ${ }^{1}$ Department of Obstetrics and Gynecology, Hurley Medical Center, Michigan State University, USA \\ ${ }^{2}$ College of Human Medicine, Michigan State University, USA
}

*Corresponding author: Abdelrahman Yousif, Department of Obstetrics and Gynecology, Hurley Medical Center, Michigan State University, USA

\begin{abstract}
1 in every 2500 females is born with Turner syndrome (TS), a result of chromosomal abnormalities of the $X$ chromosome. A common symptom of this syndrome is infertility due to ovarian dysgenesis. This case report will detail a spontaneous pregnancy in a 22-year-old female Gravida 1 Para 0 (G1P0) patient with mosaic Turner syndrome. At 34 weeks 0 days, she presented for her prenatal appointment with a past medical history significant for mosaic Turner syndrome diagnosed in her teens after a diagnostic evaluation for short stature, however, she reported regular menstrual cycles beginning at age 14 . She was then treated for a spontaneous pregnancy complicated by intrauterine growth restriction with abnormal dopplers, disrupted prenatal care, trichomoniasis, and marijuana use. Due to contractions occurring every one to two minutes with variable decelerations noted on fetal heart tones tracing (FHR), the patient was induced at $37 w 6 \mathrm{~d}$. She delivered a healthy male weighing $2750 \mathrm{~g}$ While this patient was able to conceive successfully without the aid of medical intervention, only $5.6 \%$ of TS patients conceive spontaneously. This case reveals the importance of having a discussion about fertility preserving measures as well as possible pregnancy comorbidities with TS patients and their families at the time of diagnosis.
\end{abstract}

\section{Introduction}

Turner syndrome (TS) is one of the most common chromosomal disorders. One in every 2500 female newborn infants has TS [1]. TS is the second most common cause of chromosomal abnormalities that results in miscarriage [2].

TS patients classically present with short stature, webbed neck, cardiovascular and renal abnormalities and gonadal dysgenesis. A majority of women with Turner syndrome develop accelerated follicular atresia that predisposes them to primary amenorrhea, premature ovarian failure and infertility later on in their lives. Several structural $X$ chromosome aberrations have been found in TS including, deletion, duplications, rings as well as translocations [3]. Fifty percent of TS patients have a complete loss of $X$ chromosome, while $25 \%$ have a partial deletion of one $X$ chromosome; $20 \%$ carry varying degrees of mosaicism, most commonly a $45, X / 46, X X$ karyotype. About $90 \%$ of women with TS who spontaneously conceive have a mosaic karyotype [1]. Therefore, for mosaic TS patients who experience spontaneous puberty, timely fertility counseling and exploration of fertility preservation (FP) options are crucial. In this article we report a case of spontaneous pregnancy in a patient previously diagnosed with Turner syndrome.

\section{Case}

A 22-year-old female patient G1P0 presented to our outpatient clinic for an initial prenatal visit at $34 \mathrm{wk} 0$ days after she moved from out of state, where she had most of her limited prenatal care. Years earlier, our patient was worked up for short stature at age 14 when her peripheral blood samples showed the mosaic karyotype of Turner syndrome. The patient also reported regular menstrual cycles that last 4 days with mild to moderate flow, and menarche at 14-years-old.

The patient's pregnancy was spontaneous, and its

\footnotetext{
Citation: Yousif A, Wheatley M, Abuzeid MA (2020) A Case Report of Spontaneous Pregnancy in a Mosaic Turner Syndrome Patient. Obstet Gynecol Cases Rev 7:181. doi.org/10.23937/23779004/1410181

Accepted: November 02, 2020: Published: November 04, 2020

Copyright: (C) 2020 Yousif A, et al. This is an open-access article distributed under the terms of the Creative Commons Attribution License, which permits unrestricted use, distribution, and reproduction in any medium, provided the original author and source are credited.
} 
course was affected by marijuana use, anemia, trichomoniasis infection, lapse in her prenatal care from 13 weeks to 26 weeks, and intrauterine growth restriction with abnormal Dopplers. Routine ultrasound revealed an estimated fetal weight of $10 \%$ with abnormal Doppler waves for which the patient was followed up with twice weekly nonstress tests (NSTs), ultrasound and biophysical profile (BPP). With the maternal Turner syndrome, fetal echocardiography was done to rule out cardiac anomalies and came back negative. At her last prenatal visit, the patient had frequent contractions for which she was* transferred to triage where she was found to have contractions every one to two minutes with two variable decelerations. Later on, the decision was made to start her induction of labor via vaginal prostaglandins followed by oxytocin at 37 weeks and 6 days. Afterward the patient delivered a healthy $2750 \mathrm{~g}$ male baby with Apgar scores of 8 and 9 at 1 and 5 minutes respectively.

\section{Discussion}

It has been found that infertility is a major concern for patients with TS. Considering the challenging nature of sharing information about the high likelihood of future infertility with the patients themselves and their parents, it is unsurprising that parents often feel they have inadequate knowledge to discuss fertility with their daughters. This feeling is compounded by the social stigma of infertility, desire for their daughter to have biological children, and their own loss of not having a biological grandchild [4]. These challenges make it even more important for medical providers to partner with parents and facilitate fertility-related discussions as part of TS patients' routine care [5].

There are different fertility preservation (FP) techniques, i.e., vitrification of mature oocyte after controlled ovarian stimulation, or ovarian tissue cryopreservation (OTC). Vitrification of mature oocyte is still the preferred method of FP; however, OTC is commonly used in pre-pubertal girls. In OTC, hundreds or thousands of follicles and oocytes are retrieved, via a laparoscopic approach, and frozen for later transplantation [6].

In vitro fertilization (IVF) is the most commonly used method in patients with TS. However, our patient had spontaneously conceived. Patients with Turner syndrome who have functional ovaries are considered good candidates for autologous IVF. Autologous IVF is when oocytes are retrieved and transferred back to the same patient's uterus. Mosaic peripheral blood karyotype, normal serum follicular stimulating hormone (FSH) and anti-Mullerian hormone (AMH) levels, and spontaneous puberty are markers for a successful autologous IVF [7].

After our patient encounter and with the possible premature ovarian failure sequalae, we stress the importance of timely counseling about different fertility preservation modalities in patients with established Turner syndrome diagnosis. However, pregnancy in patients with Turner syndrome is not only challenging but can be a grave prognosis as well. TS patients are at great risk of aortic dissection and rupture, such conditions should be monitored closely in those who plan to attempt pregnancy. TS patients, regardless of their karyotype, are at risk of pregnancy related morbidities and mortality, even if they show normal cardiovascular workup i.e. echocardiography or cardiac MRI. We encourage an open discussion with TS patients and their support system detailing all appropriate fertility preservation options as well as counseling on all risks and complications that might develop during pregnancy and after delivery [8].

\section{References}

1. Folsom LJ, JS Fuqua (2015) Reproductive issues in women with turner syndrome. Endocrinol Metab Clin North Am 44: 723-737.

2. Gug C, Ratiu A, Navolan D, Dragan I, Groza I-M, et al. (2019) Incidence and spectrum of chromosome abnormalities in miscarriage samples: A retrospective study of 330 Cases. Cytogenet Genome Res 158: 171-183.

3. Viuff M, Anne S, Morten MN, Simon C, Claus HG (2019) Epigenetics and genomics in turner syndrome. Am J Med Genet C Semin Med Genet 181: 68-75.

4. Wasserman D, A Asch (2012) Reproductive medicine and turner syndrome: Ethical issues. Fertil Steril 98: 792-796.

5. Reindollar R (2011) Turner syndrome: Contemporary thoughts and reproductive issues. Semin Reprod Med 29: 342-352.

6. Oktay K, Giuliano B, Karen B, Richard B, Banafsheh K, et al. (2016) Fertility preservation in women with turner syndrome: A comprehensive review and practical guidelines. $J$ Pediatr Adolesc Gynecol 29: 409-416.

7. Jacqueline K Hewitt, Yasmin Jayasinghe, David J Amor, Lynn H Gillam, Garry L Warne, et al. (2013) Fertility in turner syndrome. Clin Endocrinol 79: 606-614.

8. Practice Committee of the American Society for Reproductive Medicine (2012) Increased maternal cardiovascular mortality associated with pregnancy in women with turner syndrome. Fertility and Sterility $97:$ 282-284. 